\title{
The Phosphocreatine-ATP Energy Shuttle and Muscle Action: Misconceptions, Oversights and Insights
}

\author{
Sosale Chandrasekhar ${ }^{1 *}$ \\ ${ }^{1}$ Department of Organic Chemistry, Indian Institute of Science, Bangalore 560012 , \\ India.
}

Author's contribution

The sole author designed, analyzed and interpreted and prepared the manuscript.

Article Information

DOI: $10.9734 / / J B C R R / 2017 / 34643$

Editor(s):

(1) Yi-Ren Hong, College of Medicine, Kaohsiung Medical University, Taiwan.

Reviewers:

(1) Valentina Saccone, IRCCS Fondazione Santa Lucia, Italy. (2) Ashraf Ramadan Hafez, Deraya University, Egypt.

(3) Demarquoy, Université de Bourgogne, France.

Complete Peer review History: http://www.sciencedomain.org/review-history/19848

Opinion Article

Received $3^{\text {rd }}$ June 2017

Accepted $30^{\text {th }}$ June 2017

Published $4^{\text {th }}$ July 2017

\section{ABSTRACT}

The role of phosphocreatine (PC) as an 'energy buffer' in muscular contraction needs to be reassessed from several viewpoints. In particular, the view that the hydrolysis of PC is more exergonic than that of ATP is erroneous, as the enthalpy and Gibbs free energy changes (respectively) have generally been compared. (Apparently, the reactions are about equally exergonic.) Even so, the question of how PC levels build up during the rest phase at the expense of ATP remains. Also, in a general sense, the need for $P C$ is intriguing in itself, as its presence can be obviated by maintaining sufficient levels of ATP. Plausible explanations are proposed for these apparent conundrums.

Keywords: Actin; ATP; creatine; muscle; myosin; phosphocreatine; phosphoryl transfer. 


\section{INTRODUCTION}

\subsection{Energy Conversions in Biological Systems}

Living systems are quintessentially characterized by transformations of energy with the consequential performance of work. This thermodynamic truism is manifested in its most general sense by the involvement of the socalled high energy compounds, as typified by adenosine triphosphate (ATP, 1, Fig. 1). Whilst the ultimate source of the energy is the oxidation of foodstuffs, the mechanisms by which immediate energy needs are met in localised phenomena are complex and often convoluted.

It is, of course, generally recognized that ATP functions as an intermediate "energy currency" that facilitates the transfer of chemical potential energy, thus enabling the performance of the work that maintains living systems [1-4]. ATP is generally produced by the oxidation of foodstuffs, primarily via glycolysis and the tricarboxylic acid (Krebs) cycle, in a fascinating series of reactions employing unstable intermediates to relay the energy that ends up in ATP.

Thus, glycolysis begins by consuming 2 molecules of ATP, although producing 4 further molecules of ATP (for a net gain of 2 ATP per molecule of glucose oxidized). In contrast to this "substrate-level phosphorylation", the tricarboxylic acid cycle produces up to 11 molecules of ATP via the oxidation of $\mathrm{NADH}$ ("oxidative phosphorylation"). (ATP is also produced via "photophosphorylation" in photosynthesis).

It is believed that the hydrolysis of ATP to adenosine diphosphate (3, ADP, cf. 1) and inorganic phosphate $\left(P_{\mathrm{i}}\right)$ can yield $5-10 \mathrm{kcals} / \mathrm{mol}$ of energy, under physiological conditions [5-8]. However, ATP hydrolysis is complex, as it involves various competing ionic equilibria, and the amount of energy released depends on the conditions employed. And intriguingly, the role played by ATP in metabolic processes appears controversial and not always clear [9].

For instance, protein synthesis would be per se exergonic, although ATP is employed in the formation of the highly resonance-stabilized peptide bonds. (ATP likely performs an activating function in these reactions.) All the same, the biosynthesis of ATP and its catabolic transformations imply that the transfer of the phosphoryl group is key to the energy transfer operations that sustain living systems. This then raises the question about the existence of alternative compounds with analogous properties to those of ATP.

Indeed, one such compound, phosphocreatine $(P C, 2)$, plays a critical role in muscle action, which enables the defining characteristic of locomotion particularly among the vertebrates [1$4,10,11]$. It is currently believed that muscle contraction is driven by the hydrolysis of ATP, although the initial phase is sustained by PC via

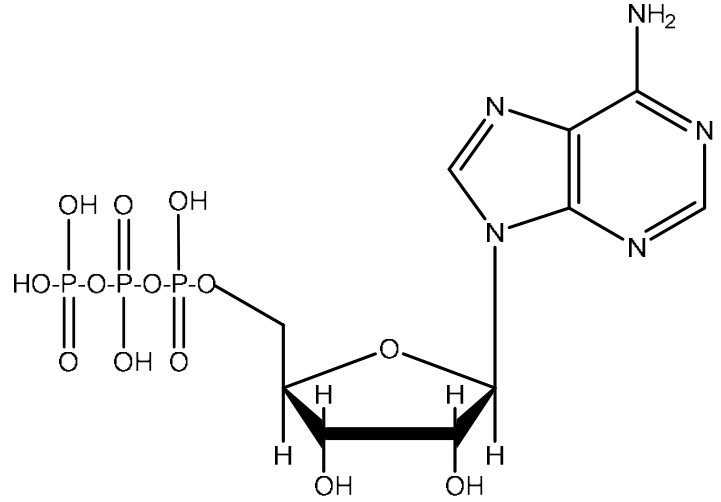

1<smiles>CN(CC(=O)O)/C(N)=N\[P+](=O)[O-]</smiles>

2

Fig. 1. Adenosine triphosphate (ATP, 1) and phosphocreatine (PC, 2) in their neutral, unionized forms 
phosphorylation of the adenosine diphosphate (ADP) by-product. This serves to replenish the levels of ATP, until its catabolic synthesis picks up (vide supra). Once the muscle action stops, the PC concentration recovers to its original resting levels via the reversal of the equilibrium involving creatine (4,cf. 2), ATP (1), PC (2) and $\operatorname{ADP}(\mathbf{3}$, Fig. 2). The reactions in this "energy shuttle" are catalyzed by the enzyme creatine kinase [12].

Hence, PC functions as an initial energy reserve that "kick-starts" muscle action. This is enabled by the presence of substantial levels of $\mathbf{4}$ and PC in (skeletal) muscle tissue. Thus, the reserve of PC allows for considerably lower levels of ATP in muscle tissue to be maintained. It is noteworthy that the hydrolysis of both $\mathbf{1}$ and $\mathbf{2}$ are apparently driven by the release of inorganic phosphate $(\mathrm{Pi})$, implying that $\mathbf{1}$ and $\mathbf{2}$ are essentially carriers of (bound) $\mathrm{Pi}$. (Creatine is produced endogenously so is not an essential nutrient, although creatine supplements have been used to enhance physical endurance and athletic performance).

Despite impressive advances in our understanding of the above phenomena, however, several questions remain to be addressed. Two, in particular, bear mention at this stage. Firstly, the relative stabilities of PC and ATP are critical to understanding how PC functions as a reserve that is also able to generate ATP (seemingly implying a contradiction between stability and reactivity). Secondly, the presence of PC itself is intriguing, as it raises the question why higher levels of ATP itself cannot be maintained in muscle tissue. These and related topics are addressed below from an essentially physicochemical perspective.

\subsection{Thermodynamic Definitions}

In the following discussion, the Gibbs free energy $(G)$, the enthalpy $(H)$ and the entropy $(S)$ are related as in Eqn. 1, $T$ being the absolute temperature [13]. The standard Gibbs free energy change $\left(\Delta G^{\circ}\right)$ is related to the equilibrium constant $(K)$ as in Eqn. 2, $R$ being the gas constant. Eqn. 3 defines the $K$ for the reaction between creatine and ATP (Fig. 2).

$$
\begin{aligned}
& G=H-T S \\
& \Delta G^{\circ}=-R T n K \\
& K=([P C][\text { ADP }]) /([\text { Creatine }][\text { ATP }])
\end{aligned}
$$

\section{DISCUSSION}

\subsection{ATP, PC and the Equilibrium between Them}

\subsubsection{Relative stabilities of ATP and PC}

Interestingly, almost a century separates the discovery of creatine (1832) and of PC (1927). The discovery of ATP too dates to 1929, indicating the arrival of high energy phosphorylated compounds on the chemicalbiology scene around a century ago. This clearly paved the way for the recognition of the key role these compounds played in metabolic processes, and for the evolution of the physicochemical underpinning of living systems.

A thermodynamic study of the hydrolysis of PC was indeed reported soon after its discovery [14], and was followed by several other determinations [15-17]. These generally referred to the reaction enthalpy $(\Delta H)$ for which, apparently, a value of $\sim 10.5 \mathrm{kcals} / \mathrm{mol}$ is currently accepted. The 1960 study of Gellert and Sturtevant [16], however, also refers to the Gibbs free energy of the hydrolysis of PC, although without arriving at a precise value.

The earlier study of Noda and co-workers [15], in fact, reports a wealth of experimental data on the PC-ATP interconversion (Fig. 2). Thus, the equilibrium constants $(K)$ are highly dependent on a variety of factors, particularly $\mathrm{pH}$ and the concentration of magnesium ions. All the same, a value of $K \sim 1$ has apparently found favour [16], which indicates that the Gibbs free energy changes $(\Delta G)$ for the hydrolysis of PC and ATP

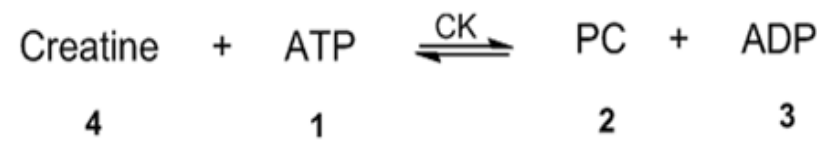

Fig. 2. The reversible reaction of creatine $(4, \mathrm{cf} .2)$ with ATP (1) to yield phosphocreatine (PC, 2) and adenosine diphosphate (ADP, 3, cf. 1), catalyzed by creatine kinase (CK) 
are nearly identical. Since the $\Delta H$ value for the hydrolysis of ATP is lower than that of PC, it is implied that the hydrolysis of $P C$ is accompanied by a considerable negative entropy change $(\Delta S)$ relative to that of ATP. (A value of $\sim-13$ e.u. was thus indicated for the $\Delta \Delta S[16])$.

It is noteworthy that previous treatments [1-4,11] have compared the $\Delta H$ for the hydrolysis of $\mathrm{PC}$ with the $\Delta G$ for the hydrolysis of ATP. This has given rise to the misconception that the hydrolysis of PC is more exergonic (by $\sim 3$ $\mathrm{kcals} / \mathrm{mol}$ ) than that of ATP, with an equilibrium constant for the PC-ATP interconversion of $~ 160$ in favour of ATP $(1 / K$, Eqn. 3, at normal temperatures). This apparently implies that PC possesses more energy than ATP, so PC is able to function as an energy reserve.

This raises the question of how a higher energy compound can accumulate during periods of rest (but vide infra). In fact, the high equilibrium constant value assumed for the PC-ATP conversion $(\sim 160$, vide supra) indicates extraordinarily high relative concentrations of creatine in muscle tissue: since the observed $\mathrm{PC} / \mathrm{ATP}$ ratios $\sim 10$, the creatine/ADP ratio would be $\sim 10^{3}$ (suggesting tens of grams of creatine per kilogram of muscle, cf. Eqn. 3)!

\subsubsection{Evolutionary implications}

As noted above, the above ideas are highly inaccurate as they are not based on a consideration of $\Delta G$ values [16]. In fact, that the equilibrium constant for the PC-ATP interconversion $\sim 1$ would be eminently reasonable in evolutionary terms. Indeed, if PC were more reactive a part of the energy would be released into the surrounding medium and thus wasted, in its conversion to ATP (molecule per molecule). Thus, the reactivities of PC and ATP have been closely matched, apparently, in the interests of energy efficiency!

\subsection{The Mechanism of Energy Transduction and Its Thermodynamic Basis}

\subsubsection{The skewed equilibrium and its profound significance}

All the same, however, the PC-ATP equilibrium (Fig. 2) is apparently maintained in favour of PC by a greater concentration of creatine present in muscle (relative to ADP). (The levels of PC are believed to be at least an order of magnitude greater than those of ATP, in resting muscle.) Still, it is intriguing that the PC-ATP equilibrium is thus weighted in favour of PC: the skewed equilibrium implies that the major part of the energy in the PC-ATP shuttle is trapped in PC!

Part of the answer to this apparent riddle, of course, is that the ATP levels are replenished catabolically (glycolysis, etc.), this being the main source of energy for the ATP synthesis. Thus, $P C$ is meant to serve as a temporary and immediate store of energy, before the catabolic processes take over. Intriguingly, again, the skewed equilibrium serves to maintain the resting ATP levels way below the PC levels, but why? In fact, this seemingly simple question may well have profound implications for our understanding of muscle action.

\subsubsection{Muscle contraction and phosphoryl} transfers: A touch of philosophy!

Skeletal muscle apparently possesses a complex fibrous structure, essentially composed of interdigitated thick and thin filaments [10]. The thick filaments are made up of the globular protein myosin, and the thin filaments of the protein actin. Interestingly, myosin can catalytically hydrolyse ATP, and is thus believed to be the seat of the process of transduction, the process (in this case) by which the energy from ATP hydrolysis is converted into the work of muscular contraction. (It is noteworthy that transduction is irreversible, in contrast to the chemical equilibrium based conversions in which ATP is normally involved [1-9]).

Thus, the key to understanding muscle action lies in the manner in which the energy from ATP hydrolysis is harnessed for the work of locomotion. ATP itself appears to be a nodal point for channelling the energy that it receives (Fig. 3), whether from catabolism or from PC via phosphoryl transfer, towards work (via hydrolysis of ATP itself).

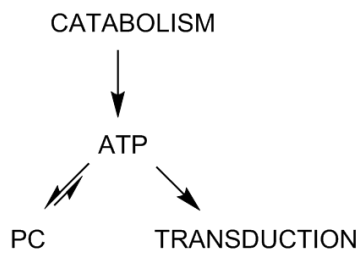

Fig. 3. ATP (1) as a nodal point involving catabolism (glycolysis, etc.), the PC-ATP shuttle and transduction leading to muscle contraction 
In fact, it is believed that smaller fibrous structures called myofibrils contract in response to the energy released from ATP. Furthermore, these processes are apparently triggered by the arrival of a nervous impulse, with calcium ions being critically involved in the event. (It is noteworthy - with a touch of philosophic fascination - that the ideas of will and action are therefore inevitably involved in the process of muscle action!).

To return to the question of the skewed equilibrium between PC and ATP: it appears the sole purpose of this is to maintain ATP levels at vanishingly low levels! Of course, this is amazing in view of the critical role played by ATP in muscle action. What is missing in this puzzle, however, is a consideration of the mechanism by which ATP is replenished via catabolism, which indeed must occur in response to a finely-tuned and highly sensitive signal.

\subsubsection{A tailored sequence of events controls and directs muscle action}

It seems likely that a rapidly depleting concentration of ATP is just the trigger for the above signal (which overall could be highly complex). This requires that the resting ATP level be relatively low, perhaps vanishingly so. A high resting ATP concentration would take far too long to deplete itself upon the initiation of the muscle action, so that the catabolic production of ATP is not initiated in time for the work to be sustained.

This explanation also implies that the shift in the PC-ATP equilibrium lags behind the signal for initiating (catabolic) ATP synthesis, although not so slowly that the muscle action cannot be sustained in the interim period! Thus, the sequence of events appears to be: initiation of muscle contraction, consequent depletion of resting ATP below a low critical level, signalling for initiating catabolic ATP synthesis, ATP synthesis from PC, ATP release from catabolism, sustained muscle action.

Importantly, of course, PC does not directly participate in the muscle action, as it is unaffected by myosin [10]. It is thus able to function as an energy buffer, by shuttling energy back and forth to the ATP nodal point. It is highly likely that the PC levels are determined by a combination of factors, essentially relating to the time taken for the above sequence of events to reach completion. (In particular, the PC levels must be sufficient to sustain the action during the interim period).

It is also noteworthy that any catabolic overproduction of ATP - for whatever reasons would be absorbed by the shuttle as PC [1-4]. The overproduction may occur particularly if the catabolic ATP synthesis cannot be turned off immediately after the cessation of the muscle contraction. Again, this may be because of an inherent lag in the signalling mechanism, which perhaps cannot be fine-tuned to perfection. In any case, the shuttle prevents a build up of excessive amounts of ATP in resting muscle tissue, which is apparently undesirable.

\subsubsection{Why not only ATP?}

The above discussion, therefore, offers a plausible rationale for the existence of the PCATP energy shuttle. This essentially relates to the question why muscle action cannot be driven and sustained by a relatively large concentration of ATP itself, rather than the "buffered mechanism" involving PC as an energy reserve.

In particular, a difference in stability cannot be the reason for this, as ATP and PC are about equally reactive hydrolytically (as seen above). Also, the view that a larger concentration of ATP will interfere with other metabolic processes is not valid, as the PC-ATP equilibrium can be shifted anyway toward ATP. (Thus, the combined PC and ATP levels represent a large pool of energetic phosphate. And interestingly, the analogy of a battery backed-up and plugged-in electronic appliance seems rather apt!).

\section{CONCLUSIONS}

The ATP-PC energy shuttle appears to have evolved both to optimise energy utilization and as part of an intricate mechanism for the transduction of chemical energy to the kinaesthetic energy of locomotion in the vertebrates.

The optimization of energy is achieved by the near identical free energies of hydrolysis of ATP and PC, which ensures that no energy is lost to the surroundings during the interconversion of ATP and PC. (Previously, the higher enthalpy of hydrolysis of PC has been erroneously compared with the Gibbs free energy change in the hydrolysis of ATP, to arrive at the misleading view that $P C$ is more reactive than ATP). 
The energy shuttle apparently also ensures that the transduction of energy in muscular contraction occurs in a highly controlled and sequential manner, the key to which may lie in the precise timing of the signal for the initiation of the synthesis of ATP (from glycolysis, etc.). This apparently depends on the resting ATP concentration being maintained at a relatively low level, its rapid depletion then serving as the signal for the initiation of catabolic ATP synthesis. The function of PC is to sustain ATP levels in the interim period. This hypothesis offers a plausible explanation for the existence of the ATP-PC shuttle (which implies that maintaining higher resting ATP levels is not a feasible option).

\section{COMPETING INTERESTS}

Author has declared that no competing interests exist.

\section{REFERENCES}

1. Nelson DL, Cox MM. Lehninger principles of biochemistry. $6^{\text {th }}$ ed. New York: WH Freeman; 2013.

2. Berg JM, Tymoczko JL, Stryer L. Biochemistry. $6^{\text {th }}$ ed. New York: WH Freeman; 2007.

3. Moran LA, Horton HR, Scrimgeour KG, Perry MD. Principles of biochemistry. $5^{\text {th }}$ ed. Boston: Pearson Education, Inc.; 2012.

4. Rawn DJ. Biochemistry. New Delhi/ Bangalore: Panima Publishing Corporation; 2004.

5. Alberty RA. Thermodynamics of the hydrolysis of adenosine triphosphate. J. Chem. Educ. 1969;46:713-719.

6. Phillips RC, George P, Rutman R. J. J. Biol. Chem. 1969;244:3330-3342.
7. Alberty RA, Goldberg RN. Standard thermodynamic formation properties for the adenosine 5'-triphosphate. Bioichemistry. 1992;31:10610-10615.

8. Iotti S, Sabatini A, Vacca A. Chemical and biochemical thermodynamics: From ATP hydrolysis to a general reassessment. J. Phys. Chem. B 2010;114:1985-1993.

9. Chandrasekhar S. Chemiosmosis and microscopic reversibility: Irreconcilable. Thermodynamic limitations of coupled equilibria and the role of ATP. vixra e-print archive; 2002.

Available: http://vixra.org/abs/1403.0022

10. Gould JL, Keeton WT. Biological science. $6^{\text {th }}$ ed. New York: WW Norton \& Co.; 1996.

11. Wyss M, Kaddurah-Daouk R. Creatine and creatinine metabolism. Physiol. Rev. 2000; 80:1107-1213.

12. Schlattner $U$, Tokarska-Schlattner $M$, Wallimann T. Mitochondrial creatine kinase in human health and disease. Biochim. Biphys. Acta. 2006;1762:164-180.

13. Atkins PW. Physical chemistry. $5^{\text {th }}$ ed. Oxford: Oxford University Press; 1995.

14. Meyerhof O, Schulz W. Über die Energieverhältnisse bei der enzymatischen Milchsäurebildung und der Synthese der phosphagene. Biochem. Z. 1935;281:292305.

15. Noda L, Kuhy SA, Lardy HA. Adenosine triphosphate-creatine transphosphorylase IV. Equilibrium studies. J. Biol. Chem. 1954;210:83-95.

16. Gellert M, Sturtevant JM. The enthalpy change in the hydrolysis of creatine phosphate. J. Am. Chem. Soc. 1960;82: 1497-99.

17. Woledge RC, Reilly PJ. Molar enthalpy change for hydrolysis of phosphorylcreatine under conditions in muscle cells. Biophys. J. 1988;54:97-104.

(c) 2017 Chandrasekhar; This is an Open Access article distributed under the terms of the Creative Commons Attribution License (http://creativecommons.org/licenses/by/4.0), which permits unrestricted use, distribution, and reproduction in any medium, provided the original work is properly cited. http://sciencedomain.org/review-history/19848 